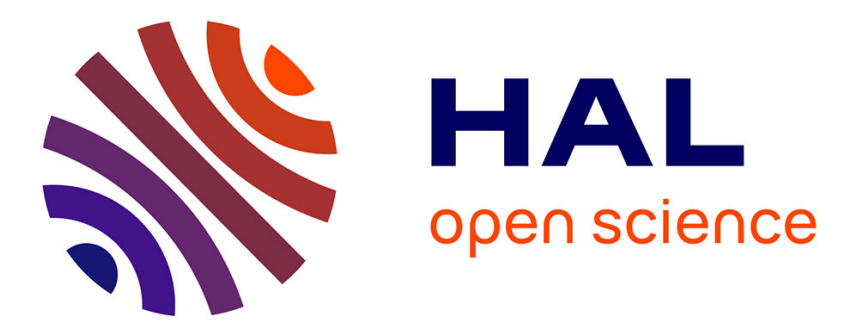

\title{
A Game Theoretical study of Peering vs Transit in the Internet
}

Giovanni Accongiagioco, Eitan Altman, Enrico Gregori, Luciano Lenzini

\section{To cite this version:}

Giovanni Accongiagioco, Eitan Altman, Enrico Gregori, Luciano Lenzini. A Game Theoretical study of Peering vs Transit in the Internet. 6th IEEE INFOCOM International Workshop on Network Science for Communication Networks (NetSciCom 2014), Apr 2014, Toronto, Canada. pp.783-788, 10.1109/INFCOMW.2014.6849330 . hal-00950851

\section{HAL Id: hal-00950851 https://hal.inria.fr/hal-00950851}

Submitted on 23 Feb 2014

HAL is a multi-disciplinary open access archive for the deposit and dissemination of scientific research documents, whether they are published or not. The documents may come from teaching and research institutions in France or abroad, or from public or private research centers.
L'archive ouverte pluridisciplinaire HAL, est destinée au dépôt et à la diffusion de documents scientifiques de niveau recherche, publiés ou non, émanant des établissements d'enseignement et de recherche français ou étrangers, des laboratoires publics ou privés. 


\title{
A Game Theoretical study of Peering vs Transit in the Internet
}

\author{
Giovanni Accongiagioco*, Eitan Altman ${ }^{\dagger}$, Enrico Gregori ${ }^{\ddagger}$ and Luciano Lenzini ${ }^{\S}$ \\ *IMT Institute for Advanced Studies, Lucca, Italy giovanni.accongiagioco@imtlucca.it \\ ${ }^{\dagger}$ INRIA, Sophia Antipolis, France eitan.altman@inria.fr \\ ${ }^{\ddagger}$ Institute of Informatics and Telematics, Italian National Research Council, Pisa, Italy enrico.gregori@iit.cnrit \\ §Information Engineering Department, University of Pisa, Italy l.lenzini@iet.unipi.it
}

\begin{abstract}
We propose a model for network optimization in a non-cooperative game setting with specific reference to the Internet connectivity. We refer to the general model shown in internal report [1], where Autonomous Systems (AS) decisions on link creation and traffic routing are strategically based on realistic interconnection costs, keeping into account the peering/transit dichotomy. Equilibria existence and convergence results were obtained in [1] only for a specific toy problem, while here we study larger scale scenarios which better fit the complex nature of the Internet. We are able to show that equilibria existence and convergence properties still hold for many possible generalizations, yet not all of them, and provide a specific example for which the system enters in a never-ending oscillation. Thanks to the use of simulations we covered those scenarios for which analytic results could not be obtained, thus analyzing a broad variety of general cases which were not studied in [1]. Simulation shows that the system, in the vast majority of cases, converges to an equilibrium. Very interestingly, even in asymmetric scenarios the equilibrium reached suggests that players tend to be symmetric with respect to the peering exchange points and send their asymmetric traffic quota via the transit service providers.
\end{abstract}

Index Terms-Internet Modeling, Complex Networks, Game Theory, AS-level Internet Topology, Supermodular Games

\section{INTRODUCTION}

The Internet consists of thousands of Autonomous Systems (AS), independently administered networks that dynamically connect together to provide end-to-end reachability. Depending on their importance and the offered services, ASes can be sorted in different tiers and categories: content providers, access providers, transit providers and so on [2]. During the past century, Internet's structure was predominantly hierarchical, with customer ASes paying their providers to carry their traffic, and the latters providing default gateways to reach any requested destination (e.g. an access provider offers network connectivity to the end users, and pays a transit provider to use it as a gateway). This kind of relationship is known as "customer to provider" or transit, and the pricing strategy of providers w.r.t. their customers is typically volume-based [3].

Nowadays Internet, as reported in [4], is evolving from its previous structure and becoming flatter through the introduction of peering meshes, with ASes establishing bilateral agreements to exchange traffic between them for free. This

This work has been partially supported by the European Commission within the framework of the CONGAS project FP7-ICT-2011-8-317672.

The first author would like to thank Alexandre Reiffers for the interesting discussions on game theoretic modeling aspects. kind of relationship is known as "peering", and in this case the cost of each AS consists of laying out and maintaining the physical interconnection. In order to avoid exploits, the peering ASes must agree on some policy, which typically forbids the use of each other as default gateway, balances the traffic ratio and so on [5]. The massive presence of peering meshes would be impossible if ASes had to lay out physical interconnections one by one, and was achieved thanks to the increasing deployment of Internet Exchange Points (IXPs) [6]. These public interconnection points can be roughly compared to large peering hubs: when an AS joins an exchange point, it can peer with a subset (or even all) the other ASes which participate to the same IXP. In order to connect to an IXP, customers typically pay a flat, monthly based, fee, which depends on the cost for maintaining the IXP equipment, in proportion to how much they use it: this means that maintainance costs are shared among all the participants of an exchange point [7]. Please note that the addition of new customers can lower the costs of a single participant, therefore this cost function is quite different from standard modelization, like the one done in [8].

ASes joining the Internet face the complex question of what is the best strategy for offering their services (e.g. traffic delivery) at the lowest possible cost. While the answer was easy in the past century, due to the existence of transit as the unique interconnection policy, today's answer is much more complex. In fact, peering policies and IXPs brought new variables to the problem, such as the fact that the outcome of an AS decision also depends on what other ASes, dealing with the same problem, do: due to this aspect, we believe that a game-theoretical analysis of the problem would be highly insightful. While, for the sake of mathematical tractability, it is impossible to realistically model the whole decision space of an AS, the model proposed in [1] keeps into account many realistic factors like port capacity, AS reachability and realistic interconnection costs. The model focuses on access provider ASes who need to set up their connections and route traffic, and highlights the fundamental difference between transit and peering interconnection policies. In fact, a better understanding of this problem is crucial if we really want to grasp the behavior of ASes living in the Internet ecosystem.

The main contributions of this work are twofold. First of all, we extend results presented in [1], showing the existence of equilibria and an algorithm converging to them even for 
generalized version of the game therein proposed. Moreover, we use simulation to show that the main findings of [1], like the emerging competition (first observed in [9]) between the two facilities enabling either transit or peering connectivity, still hold for more general cases. Even more interestingly, we notice that also for asymmetric scenarios, the equilibrium reached suggests that players tend to be symmetric with respect to the internet exchange points and send their asymmetric traffic quota via the transit service providers.

The remainder of this paper is organized as follows: in Section II we describe the related work, Section III recalls the general model, in Section IV we give convergence results for several scenarios, in Section V we simulate the system's behavior for more general cases, then conclude in Section VI.

\section{RELATED WORK}

The majority of Internet AS-level topology models are based on graph theory and try to reproduce topological properties observed in the Internet's measured graph, such as its powerlaw degree distribution [10], the small-world property [11] and other structural properties (communities, cliques, etc..) [12]. In order to generate graphs [13] which exhibit such properties, these kind of models either define attachment criteria, as in [11], [14], [15], [16] or solve constrained optimization problems (see [17], [18]).

The approach of [1] is quite different, since it tries to understand how connections are formed as the result of a game between ASes. In this context, the realm of network formation games (see [19], [20]) investigates existence and properties of equilibria in a network created by rational players, each one with their costs and utility functions. Unfortunately, at the present stage, these models cannot be used to study Internet or any other real life network, due to the simplifications needed for mathematical tractability. Taking a different approach, agent-based computational models such as GENESIS [21], include more realistic considerations and simulate the dynamic of network formation. These models however, are analytically intractable and it is impossible to give convergence guarantees.

Given all the difficulties arising when trying to model the whole network formation process, [1] prefers to analyze the decision problem faced by ASes connecting to a pre-existing network, in order to deliver traffic according to some demands. Authors of [22] present a game-theoretic modelization of a network where access providers optimize their costs when connecting to a subset of content provider. The assumptions made for the cost functions, however, do not take into account the differences between transit and peering interconnection policies, as the work concentrates more on the analysis of network-neutrality issues. An interesting analysis on peering economics and private internet exchanges is made in [23]. Unfortunately the "private peering" therein analyzed is quite different, both in rules and costs, from the "public peering" brought by IXPs, and is unable to explain nowadays phenomenons like the peering meshes (see the Introduction). In order to investigate the present-state of peering the model in [1] explicitely introduces the concept of exchange point.

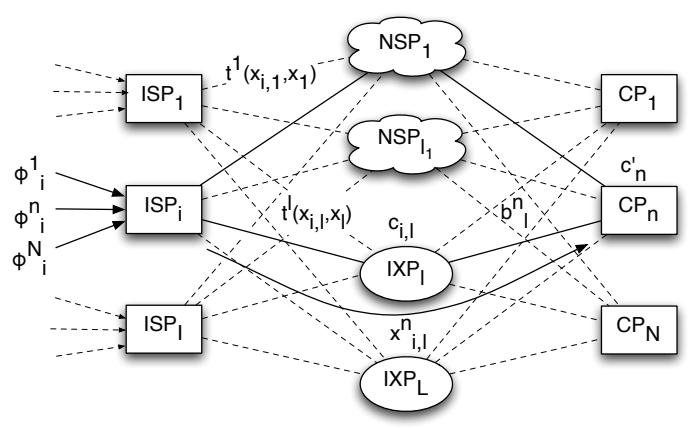

Figure 1. General Model

\section{General Scenario}

In the following we briefly describe the general model proposed in [1]. The reader can refer to section III of that report for a comprehensive dissertation.

\section{A. Description}

First of all, ASes have been categorized according to what follows (as also done by PeeringDB [24]):

Internet Service Provider $(I S P)^{1}$ : access provider giving to the end users a way to reach the Internet and its contents.

Content Provider $(C P)^{1}$ : which has physical access to the contents users are looking after.

Internet Exchange Point $(I X P)^{1}$ : a facility that provides peering connection to all its participants, in order to reach every CP connected to the IXP.

Network Service Provider $(N S P)^{1}$ : a facility providing transit connection to its customers, in order to reach every possible CP.

The general network has $i \in\{1, \ldots, I\}$ ISPs, $n \in\{1, \ldots, N\}$ CPs and $l \in\{1, \ldots, L\}$ transmit facilities (TF), which can be either NSPs or IXPs. Without loss of generality, it is supposed that TFs $j_{1} \in\left\{1, \ldots, l_{1}\right\}$ are NSPs, while TFs $j_{2} \in\left\{l_{1}+\right.$ $1, \ldots, L\}$ are IXPs. Each ISP $i$ has a demand for a CP $n$, indicated as $\phi_{i}^{n}$. The players of the game are the ISPs, which need to decide how to split their demand among all possible transmit facilities, as shown by figure 1 .

Variable $x_{i, l}^{n}$ indicates the flow from ISP $i$ to CP $n$ through $\mathrm{TF} l$, therefore the strategy of ISP $i$ is given by the vector $\mathbf{x}_{\mathbf{i}}=\left(x_{i, 1}^{1}, \ldots, x_{i, l}^{n}, \ldots, x_{i, L}^{N}\right) \in \mathbb{R}^{L \times N}$, while the strategy of all the other players is expressed as $\mathbf{x}_{\backslash \mathbf{i}} \in \mathbb{R}^{(I-1) \times L \times N}$. The goal of each player is to serve, at the minimum possible cost, his demand $\phi_{\mathbf{i}}=\left(\phi_{i}^{1}, \ldots, \phi_{i}^{N}\right) \in \mathbb{R}^{N}$ by splitting it into several flows $x_{i, l}^{n}$. The cost of player $i$ is given by the summation of three costs:

TF usage cost : which depends on the transmit facility (NSP or IXP) used. As shown in [1], the NSP usage cost is linear in the amount of flow that each player sends to it:

$$
a_{l} \sum_{n} x_{i, l}^{n} \quad l \leq l_{1}
$$

where $a_{l, l \leq l_{1}}$ represents the transit price of NSP $l$ per unit of flow. The IXP participation cost, as previously said and furtherly clarified in [1], depends on the cost for maintaining

\footnotetext{
${ }^{1}$ ISPs, NSPs and CPs are typically ASes. IXPs are not ASes, even if their infrastructure is under a single administrative control.
} 
the IXP equipment, in proportion to how much each player uses it, therefore it can be written as:

$$
\frac{\sum_{n} x_{i, l}^{n}}{\sum_{i} \sum_{n} x_{i, l}^{n}} h_{l}\left(x_{l}\right) \quad l>l_{1}
$$

where $h_{l}$ represents the maintenance cost of IXP $l$ as a function of the total flow through the IXP. As detailedly shown in [1], the mantainance cost can be approximated with that of ports, which is shown to be fitted by a square root: $h_{l}\left(x_{l}\right)=a_{l} \sqrt{\sum_{i} \sum_{n} x_{i, l}^{n}}$, where $a_{l, l>l_{1}}$ is a constant relating the total flow through IXP $l$ with its mainteinance cost.

TF capacity cost : which arises from the fact that flow on each link between an ISP and a TF cannot exceed a fixed capacity $c_{i, l}$. With the proper assumptions, illustrated in [1], the constraint is modeled as a cost growing to infinity as the flow over the link approaches the capacity limit:

$$
\frac{1}{c_{i, l}-\sum_{n} x_{i, l}^{n}} \sum_{n} x_{i, l}^{n}
$$

Typically the capacity of an NSP can be assumed to be much larger than that of an IXP: $c_{N S P} \gg c_{I X P}$ (see [3], [7]), moreover we assume that capacities are symmetric w.r.t. the players, therefore $c_{i, l}=c_{l} \forall i$.

$C P$ reachability cost : as previously said, while NSPs can be used to reach every possible $\mathrm{CP}$, through IXPs a player can only reach the subset of CPs connected to the same IXP they participate into. If we indicate with $b_{l}^{n}$ the cost of transporting one unit of flow from $\mathrm{TF} l$ to $\mathrm{CP} n$, we can express this constraint by putting:

$$
b_{l}^{n}= \begin{cases}0 & \text { if } l \leq l_{1} \vee I X P_{l} \rightarrow C P_{n} \\ \infty & \text { otherwise }\end{cases}
$$

After some algebraic manipulations, explicitely shown in [1], the cost function of each player can be written as:

$$
\begin{aligned}
& C^{i}\left(\mathbf{x}_{\mathbf{i}}, \mathbf{x}_{-\mathbf{i}}\right)=\sum_{l} \sum_{n} x_{i, l}^{n}\left[f^{l}\left(\sum_{i} \sum_{n} x_{i, l}^{n}\right)+\right. \\
& \left.\quad+g^{l}\left(\sum_{n} x_{i, l}^{n}\right)+b_{l}^{n}\right] \\
& \begin{cases}f^{l}\left(\sum_{i} \sum_{n} x_{i, l}^{n}\right)= \begin{cases}a_{1} & l \leq l_{1} \\
\frac{a_{l}}{\sqrt{\sum_{i} \sum_{n} x_{i, l}^{n}}} & l>l_{1}\end{cases} \\
g^{l}\left(\sum_{n} x_{i, l}^{n}\right)=\frac{1}{c_{l}-\sum_{n} x_{i, l}^{n}}\end{cases}
\end{aligned}
$$

Equation (5) gives the general expression of the cost function for each player and, as already observed in [1], it is a nonconvex function of $x_{i, l}$, therefore we cannot directly establish existence of pure equilibria using general frameworks like the one described in [8].

The best response of player $i, B R_{i}\left(\mathbf{x}_{\backslash \mathbf{i}}\right)$, is obtained by both minimizing this cost function and satisfying the flow constraints, that is, the total flow served for every $\mathrm{CP}$ has to be equal to the demand $\phi_{i}^{n}$. Therefore we have:

$$
\left\{\begin{array}{l}
B R_{i}\left(\mathbf{x}_{\backslash \mathbf{i}}\right)=\arg \min _{\mathbf{x}_{\mathbf{i}}} C^{i}\left(\mathbf{x}_{\mathbf{i}}, \mathbf{x}_{\backslash \mathbf{i}}\right) \\
\text { s.t. } \quad \sum_{l} x_{i, l}^{n}=\phi_{i}^{n} \quad \forall n
\end{array}\right.
$$

The vector $\mathbf{x}^{*}=\left(\mathbf{x}_{1}^{*}, \ldots, \mathbf{x}_{\mathbf{I}}^{*}\right) \in \mathbb{R}^{I \times L \times N}$ is an equilibrium of the game if and only if $\mathbf{x}_{\mathbf{i}}^{*} \in B R_{i}\left(\mathbf{x}_{\backslash \mathbf{i}}^{*}\right) \forall i$, that is, if the strategy of each player is a best response to the strategies of other players.

\section{AnAlytic Results}

\section{A. Base Theoretical Results}

Theoretical results for the model, regarding the existence of equilibria and algorithms that converge to them, are based on the theory of supermodular games. Here we briefly review the defition of supermodular games and the main results of [1].

\section{Definition 1. Supermodular games [25]}

Consider a generic game $G$, where players' payoffs are given by an utility function $u: \mathbb{R}^{k} \rightarrow \mathbb{R}$. When $u$ is twice continuously differentiable, the game is said supermodular if the mixed second derivatives of the utility function are always positive. In the proposed model players are minimizing costs rather than maximizing utilities, therefore this kind of game is supermodular iff:

$$
\frac{\partial^{2} C(\mathbf{x})}{\partial x_{i} \partial x_{j}} \leq 0 \quad \forall i \neq j
$$

\section{Definition 2. Symmetric supermodular games [1]}

While supermodular games require property (8) to hold for every possible strategy $x_{i}$, symmetric supermodular games, as defined in [1], are those for which (8) only holds along the symmetric axis, that is when $x_{i}=x_{j} \forall i \neq j$.

Theorem 1 of [26] proves the existence of equilibria for supermodular games. It also provides a way of computing them, as it shows that due to the monotonicity of best response sequences, they converge to a limit which is shown to be an equilibrium. Along the same line of reasoning, Theorem 4 of [1] relaxes this result, showing that it holds also for symmetric supermodular games, where equilibria are given as the limit of symmetric best response sequences (defined in Section IV.A of [1]).

Starting from the general model presented in section III, [1] defines the Minimal Complexity Model (MCM), which has two ISPs, two CPs, two TFs (an NSP and an IXP), and a fully connected topology. It is shown that the MCM is a symmetric supermodular game (the proof is based on showing that (8) holds along the symmetric axis), and therefore it has at least one pure equilibrium for symmetric demands, given as the limit of a symmetric best response sequence.

While interesting on its own, as it provides a way to highlight basic differences between transit and peering policies (and shed light on the competition between NSPs and IXPs, first observed in [9]), one might argue that the MCM topology is quite small and simplified to represent the Internet. Here we explicitely tackle this problem by both extending the convergence results to more general scenarios, and using simulation to cover other cases for which we couldn't obtain analytic results, thus analyzing a broad variety of general cases which weren't covered in [1].

\section{B. Extended Results}

Some of the scenarios for which the theorems shown in the previous section did not apply where tested through simulation in [1], and the observed results brought to the conjecture that convergence was indeed a more general property. As a 
matter of fact, we are able to generalize Theorem 5 of [1], showing that symmetric supermodularity holds also for the general scenario of section III.

We consider a system with $I$ ISPs, $N$ CPs, and $L$ TFs, with $l_{1}=1$, in a possibly disconnected topology. While having multiple IXPs is fundamental for understanding how players aggregate around exchange points, especially in presence of reachability constraints (IXPs may be connected to only a subset of CPs), this is not the case for NSPs, due to the fact that their cost is independent from other players' choice, and they are necessarily connected to all possible CPs. Therefore, without loss of generality for our problem, it is safe to consider only a single NSP for $l=1$, and $L-1$, for $l=2 . . L$, IXPs as we do here. In the following we will always refer to this system, which is a single NSP version of figure 1.

The cost function of section III can thus be rewritten separating the NSP component from the IXPs, therefore:

$$
\begin{aligned}
& C^{i}\left(\mathbf{x}_{\mathbf{i}}, \mathbf{x}_{-\mathbf{i}}\right)=\sum_{n} x_{i, 1}^{n}\left(a_{1}+\frac{1}{c_{1}-\sum_{n} x_{i, 1}^{n}}\right)+ \\
& +\sum_{l \neq 1} \sum_{n} x_{i, l}^{n}\left(\frac{a_{l}}{\sqrt{\sum_{i} \sum_{n} x_{i, l}^{n}}}+\frac{1}{c_{l}-\sum_{n} x_{i, l}^{n}}+b_{l}^{n}\right)
\end{aligned}
$$

where the CP reachability cost for the NSP has been removed since we know from (4) that $b_{1}^{n}=0 \forall n$.

Theorem 3 and Corollary 4, whose complete proofs can be found in the full version [27], demonstrate existence of equilibria and convergence of the symmetric best response algorithm for the general case just formalized.

Theorem 3. The game defined in (9) is symmetric supermodular.

Sketch of the Proof: The proof is based on showing that (8) holds along the symmetric axis for any possible combination of indexes:

$$
\left.\frac{\partial^{2} C^{i}\left(\mathbf{x}_{\mathbf{i}}, \mathbf{x}_{-\mathbf{i}}\right)}{\partial x_{j, \bar{l}}^{\bar{n}} \partial x_{i, l}^{n}}\right|_{x_{i, l}^{n}=x_{j, \bar{l}}^{\bar{n}}} \leq 0 \quad \forall i \neq j, \forall l, \bar{l}, n, \bar{n}
$$

Corollary 4. The game defined in (9) has at least one pure equilibrium for symmetric demands, given as the limit of a symmetric best response sequence.

\section{Subcases Analysis}

After proving existence of equilibria for the general case, here we analyze some subcases in order to understand what kind of equilibria we should expect for specific scenarios. We have two main categories:

a) Fully Connected Topologies: Suppose that we have a fully connected topology, meaning that $b_{l}^{n}=0 \forall l, n$. In such a case, we can take the summation over $n$ and consider cumulative flows and demands:

$$
\begin{cases}x_{i, l}=\sum_{n} x_{i, l}^{n} & \text { cum. flow ISP } P_{i} \rightarrow T F_{l} \\ \phi_{i}=\sum_{n} \phi_{i}^{n} & \text { cum. demand ISP }\end{cases}
$$

We can now substitute these two variables inside cost function (5), thus obtaining an equivalent problem where the strategy of each player is a vector $\mathbf{x}_{\mathbf{i}}=\left(x_{i, 1}, \ldots, x_{i, l}, \ldots, x_{i, L}\right) \in$

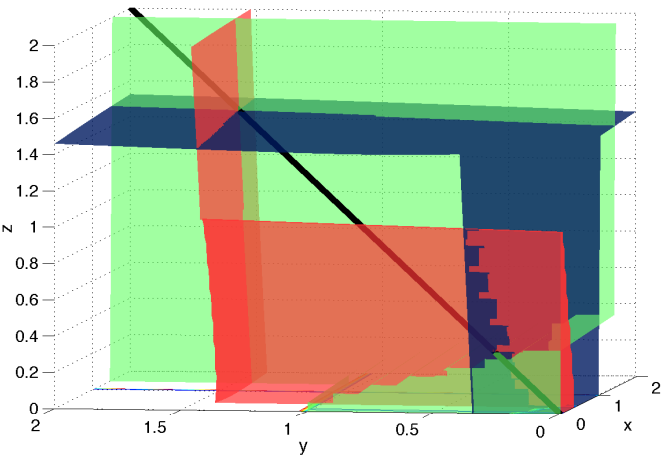

Figure 2. BRI for 3 Players: $a_{1}=2, a_{2}=2, \phi=2, c_{1}=10, c_{2}=3$ $\mathbb{R}^{L}$. This means that, in fully connected topologies, our system is equivalent to another one where we only have a single $\mathrm{CP}$, and each player has to serve a cumulative demand $\phi_{i}$ for that CP. This happens because there are no reachability constraints, therefore from a player's perspective the specific $\mathrm{CP}$ from which he has to fetch data is not relevant.

b) Symmetric IXPs : Suppose that all the IXPs have the same costs, capacities and reachability matrix: $a_{l}=a_{I X P}$, $c_{l}=c_{I X P}, b_{l}^{n}=b_{I X P}^{n} \forall l \neq 1$. Due to their symmetry, there is an equilibrium where traffic is split equally among them [28], therefore we might think of transforming this problem in an equivalent one having a single IXP with the same reachability matrix and transformed costs and capacities. Unfortunately, we were unable to perform this conversion due to the form of the cost function for the IXPs. In fact, as we see from (5) and (6), the non linear port cost $h_{l}$ makes it quite different for players to share small traffic quantities rather than large ones.

The analysis of the two categories highlights that scenarios with multiple CPs can be highly simplified with fully connected topologies, while in the case of multiple IXPs, even if symmetric, the analysis can be quite difficult. To conclude this section, we analyze in more detail the simplified case where we have fully connected topology and just one IXP. Due to the fact that we can handle the multiple CPs as if there was just a single one, this scenario is quite similar to the MCM, except that we have a generic number $I$ of players. However, thanks to Corollary 4, we now know that for symmetric demands $\phi_{i}=\phi \forall i$ we can compute equilibria as the limit of symmetric best response sequences. Just like it was done for the MCM, we can use algorithm 1 of [1] to compute the equilibria.

With the purpose of analyzing number and position of equilibria, for the case of $I=3$ (it would be difficult to represent more dimensions), we can draw the Best Response Intersection (BRI) graph, shown in Figure 2. Just like in the MCM, the picture shows three equilibrium points, obtained by the intersection of the three surfaces representing the players' best responses. As we see from the straight line crossing all such points, the three equilibria are symmetric, with the leftmost (traffic split between NSP and IXP) and the rightmost (all flows through the NSP) being the stable ones.

\section{Simulative Results}

In this section we show results of simulations, performed both for symmetric scenarios, for which convergence has been 


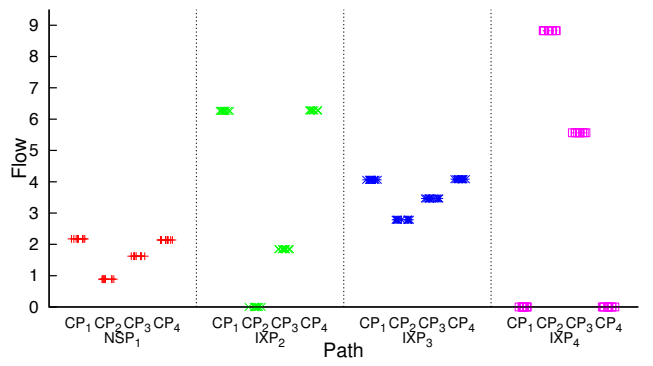

Figure 3. Symmetric Case Flows Scatterplot: $\phi_{i}^{n}=12.5$

proven in the general case, and asymmetric scenarios, for which we have no proofs. In fact, as we'll see later on, in this last case it is possible for players to never reach an equilibrium.

Simulations have been performed using the same symmetric best response algorithm shown in [1]: iteratively, each player performs its best response to the set of other players' strategies. If the simulation converges, the output is the equilibrium for the given input parameters, which are:

- the number of ISPs, TFs and CPs, respectively $I, L, N$;

- the cost function parameters $a_{l}, c_{l}, b_{l}^{n}$ and demands $\phi_{i}^{n}$. Moreover, it is important to set a startingpoint, because, as shown in [1], on startup IXPs need a critical mass, represented by a share of the total traffic in the system, in order to be able to attract players.

\section{A. Symmetric Case}

We simulate a scenario with $I=10$ ISPs, $L=4$ TFs $(L-1$ symmetric IXPs) and $N=4 \mathrm{CPs}$. The connectivity matrix is:

$$
b_{l}^{n}= \begin{cases}\infty & (l, n)=(2,2) \vee(l, n)=(4,1) \vee(l, n)=(4,4) \\ 0 & \text { otherwise }\end{cases}
$$

The cost parameter $a_{l}$ has been chosen in order to be similar to present reality. We use [3] for inferring a general transit cost of $a_{1}=a_{N S P}=3$, and [1] for the IXP cost $\forall l \neq 1 a_{l}=a_{I X P}=30$, using the fit therein obtained for the port costs of the MIX, a specific italian IXP. All users have symmetric flows $\phi_{i}^{n}=12.5 \forall i, n$ and their capacities to the facilities are $c_{1}=c_{N S P}=100$ and $\forall l \neq 1 c_{l}=c_{I X P}=20$, so that $c_{N S P} \gg c_{I X P}$.

As already happened in the MCM, depending on the startingpoint we notice the existence of multiple equilibria. In fact, if the initial condition is such that one or more IXPs are underutilized, than at equilibrium those IXPs will not be used. This phenomenon corroborates the outcomes of [1], showing that indeed even in general scenarios the competition between NSPs and IXPs, and even between IXP themselves, strongly emerges. Differently from the MCM, in this case we observe more than two stable equilibria, since any combination with one or many unused IXPs can be an equilibrium.

Suppose now that the startingpoint is such that flows are split equally among the facilities, so that all IXPs have the critical mass to attract players. Figure 3 shows the scatterplot at equilibrium. In this plot, each dot represents the flow quantity that each user sends on a given path (that is, to a fixed $\mathrm{CP}$ through a given IXP). Due to symmetry, we observe that all users will behave symmetrically on the same path, and this is

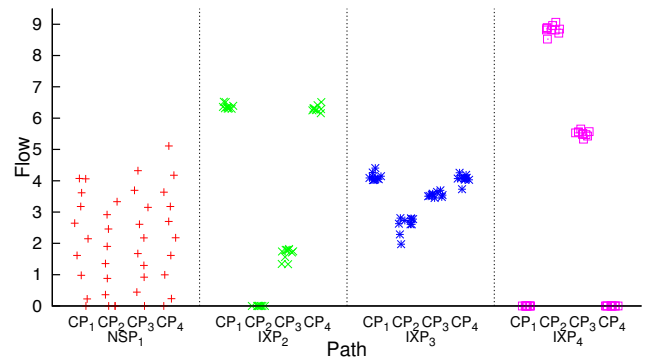

Figure 4. Asymmetric Case Flows Scatterplot: $\phi_{i}^{n}=10 \rightarrow 15$

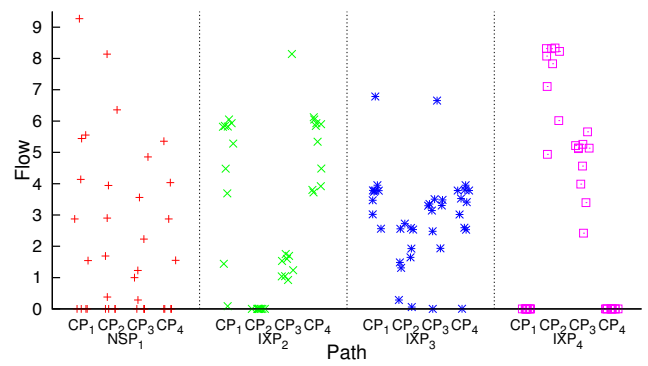

Figure 5. Asymmetric Case Flows Scatterplot: $\phi_{i}^{n}=6.5 \rightarrow 18.5$

exactly what happens in Figure 3. There is generally a low utilization of the NSP, which rises a little bit for those CPs with a worse reachability matrix $\left(C P_{1}, C P_{4}\right)$.

\section{B. Asymmetric Case}

We now show the impact of asymmetric players' demands. In this case, convergence of the best response sequence is not guaranteed by Corollary 4, however, we know that if the simulation converges we certainly reach an equilibrium [1].

We simulate a scenario with exactly the same parameters as in the symmetric case, except that now the demands grow linearly from $\phi_{1}^{n}=10 \forall n$ to $\phi_{I}^{n}=15 \forall n$. The average demand is still 12.5 , but now the demand of the last player is 1.5 times that of the first one. The scatterplot at equilibrium is shown in Figure 4. Very interestingly, even if demands are asymmetric, paths of flow tend to be almost symmetric for the IXPs, while they spread apart for the NSP. This happens because the benefits of sharing costs at the exchange points is bigger when the traffic ratio is approximately the same between participants, therefore players tend to "symmetrize" around the IXPs. Due to the fact that flows around the IXPs are more or less symmetric, players will send the traffic residual through the NSP, which will see highly asymmetric patterns.

In previous case, the asymmetry in players' demands was not very pronounced. Let's now see what happens when the demands go from $\phi_{1}^{n}=6.5 \forall n$ to $\phi_{I}^{n}=18.5 \forall n$, meaning that last player demand is nearly three times that of player one. Again, Figure 5 shows the scatterplot at equilibrium. Due to the heavily unbalanced demands, the symmetric patterns around the IXPs are still present, but much less pronounced. While in the previous case equilibrium was driven by the simple rule of "symmetric behavior", in this case the outcome is more difficult to predict. In general, due to the asymmetry, cost benefits of players' for using exchange points decrease, therefore we observe, on average, an higher quantity of flow going through the NSP. 
The phenomenons emerged through this analysis can provide some preliminary insight on how to devise optimal policies to handle peering traffic at IXPs. More specifically, the "symmetric behavior" rule highlights that it is beneficial to balance traffic as much as possible, therefore IXP owners should create few classes of traffic (namely, few different port sizes), and participants should try to aggregate traffic on these ports, since unbalanced flows must be handled at NSPs and bring to suboptimality. While simulations have been carried out with a limited network size due to computational constraints, the conclusions are fairly general, therefore we expect similar results to hold for larger-scale scenarios.

\section{Non Convergence}

Even quite simple scenarios for which we cannot apply Theorem 3, might lead to a situation where players' behavior oscillates, never reaching an equilibrium. One of such cases can be found in the full version of the paper [27]. Consider a system with two symmetric IXPs and an asymmetric starting point s.t. a group of players send more traffic to one of the IXPs and less to the other, while the other group of players do the opposite. Due to the asymmetric assumption we cannot apply Corollary 4, and simulation shows that this scenario might never reach an equilibrium. This happens when players enter a never-ending oscillation between the first and the second group, as detailedly shown in [27].

\section{CONCLUSIONS AND FUTURE WORK}

This work, starting from [1], extends the analysis therein proposed for the MCM model to more general scenarios which better fit the complex nature of the Internet. From a game theoretic perspective, we were able to prove that even the general model falls in the category of Symmetric Supermodular games, thus obtaining both existence of equilibria and convergence of symmetric best response sequences for our game. Moreover, we have shown that for asymmetric sequences convergence may fail, by explicitely simulating a specific scenario for which the system enters in a never-ending oscillation. From an engineering perspective, the contributions are twofold. First of all, we corroborate the main result of [1] by highlighting once more the competition between IXPs, providing customers the ability to lay out peering connections, and NSPs, high level providers selling transit connections. Second, we have shown that also for asymmetric cases the system often reaches an equilibrium. Even more interestingly, the equilibrium reached in asymmetric cases suggests that players tend to "symmetrize" as much as possible with respect to the peering exchange points and send their asymmetric traffic quota via the transit service providers. This observation can provide insights on how to devise optimal policies to handle peering traffic at IXPs.

The work calls for extensions in at least two directions, actively pursued at the time of writing. The first consists of an in-depth study of the properties of asymmetric cases, e.g. the analysis of their optimality using metrics like the price of anarchy, stability and fairness, already considered for the MCM. The second, taken directly from [1], follows from the dynamic nature of the Internet. In reality, both transit and peering costs can be renegotiated over time, therefore by adding as players the TFs we can analyze a dynamic game where their strategies are intertwined with that of ISPs.

\section{REFERENCES}

[1] G. Accongiagioco, E. Altman, E. Gregori, and L. Lenzini, "Peering vs transit: a game theoretical model for autonomous systems connectivity," tech. rep., [Online] Available: http://tinyurl.com/naj7o4t, 2013.

[2] W. B. Norton, The 2013 Internet Peering Playbook. 2011.

[3] W. B. Norton, "Internet transit prices - historical and projected," tech. rep., Dr. Peering White Paper, 2010.

[4] A. Dhamdhere and C. Dovrolis, "The internet is flat: modeling the transition from a transit hierarchy to a peering mesh," in Proceedings of the 6th International COnference, Co-NEXT, ACM, 2010.

[5] W. B. Norton, "A study of 28 peering policies," tech. rep., 2010.

[6] E. Gregori, A. Improta, L. Lenzini, and C. Orsini, "The impact of IXPs on the AS-level topology structure of the Internet," Computer Communications, vol. 34, pp. 68-82, Jan. 2011.

[7] W. B. Norton, "The art of peering - the ix playbook," tech. rep., Dr. Peering White Paper, 2010.

[8] A. Orda, R. Rom, and N. Shimkin, "Competitive routing in multiuser communication networks," IEEE/ACM Transactions on Networking, vol. 1, pp. 510-521, Oct. 1993.

[9] B. Ager, N. Chatzis, A. Feldmann, N. Sarrar, S. Uhlig, and W. Willinger, "Anatomy of a large european ixp," in Proc. of the 2012 ACM SIGCOMM Conference, pp. 163-174, 2012.

[10] M. Faloutsos, P. Faloutsos, and C. Faloutsos, "On Power-Law Relationships of the Internet Topology," ACM SIGCOMM Computer Communication Review, 1999.

[11] D. J. Watts and S. H. Strogatz, "Collective dynamics of 'small-world' networks.," Nature, vol. 393, pp. 440-2, June 1998.

[12] E. Gregori, L. Lenzini, and C. Orsini, "k-clique Communities in the Internet AS-level Topology Graph,” 31st International Conference on Distributed Computing Systems Workshops, pp. 134-139, June 2011.

[13] H. Haddadi, M. Rio, G. Iannaccone, A. Moore, and R. Mortier, "Network Topologies: Inference, Modeling and Generation," IEEE Communications Surveys, vol. 10, no. 2, pp. 48-69, 2008.

[14] S. Yook, H. Jeong, and A. Barabási, "Modeling the Internet's large-scale topology," in PNAS, pp. 1-15, 2002.

[15] X. Wang and D. Loguinov, "Understanding and Modeling the Internet Topology : Economics and Evolution Perspective," IEEE Transactions on Networking, vol. 18, no. 1, pp. 257-270, 2010.

[16] G. Accongiagioco, E. Gregori, and L. Lenzini, "A Structure-Based Topology Generator for the Internet' s Core," in 4th Workshop of Italian group on Quantitative Methods in Informatics (InfQ), pp. 1-8, 2013.

[17] A. Fabrikant, E. Koutsoupias, and C. H. Papadimitriou, "Heuristically optimized trade-offs: A new paradigm for power laws in the internet," in Proceedings of the 29th ICALP '02, Springer-Verlag, 2002.

[18] H. Chang, A. Arbor, P. Ave, and F. Park, "To Peer or not to Peer: Modeling the Evolution of the Internet's AS-level Topology," in Proceedings of 25th IEEE Conference on Comput. Commun., INFOCOM '06.

[19] M. Jackson, Social and Economic Networks. Princeton Univ.Press, 2010.

[20] S. Goyal, Connections: an introduction to the economics of networks. Princeton University Press, July 2007.

[21] A. Lodhi, A. Dhamdhere, and C. Dovrolis, "GENESIS: An agent-based model of interdomain network formation, traffic flow and economics," 2012 Proceedings IEEE INFOCOM, pp. 1197-1205, Mar. 2012.

[22] T. Jiménez, Y. Hayel, and E. Altman, "Competition in access to content," in Proceedings of IFIP Networking, pp. 211-222, 2012.

[23] S. Shakkottai and R. Srikant, "Economics of network pricing with multiple isps," IEEE/ACM Trans. Netw., vol. 14, Dec. 2006.

[24] "Peeringdb - http://www.peeringdb.com."

[25] D. D. Yao, "S-modular games, with queueing applications," Queueing Systems, vol. 21, pp. 449-475, Sept. 1995.

[26] E. Altman and Z. Altman, "S-modular games and power control in wireless networks," IEEE Trans. on Automatic Control, vol. 48, 2003.

[27] G. Accongiagioco, E. Altman, E. Gregori, and L. Lenzini, "A game theoretical study of peering vs transit in the internet," tech. rep., [Online] Available: http://tinyurl.com/nkdob7z, 2013.

[28] S. fen Cheng, D. M. Reeves, Y. Vorobeychik, and M. P. Wellman, "Notes on equilibria in symmetric games," in Proceedings of the 6th International Workshop GTDT, pp. 71-78, 2004. 